\section{NO-TOUCH HAND DISPENSER}

The new Saraya UD9000 compact wall mountable touch-less infrared dispenser with its 'no-touch' technology will accurately dispense Alsoft-E clinical/ surgical hand disinfectant or hand soap. It has a compact new design; dispenses from a choice of 1.2 litres of Saraya hand soap or a disinfectant sealed refill; the disinfectant allows for full clinical hand disinfection in just 15 seconds; it has a lockable key operated unit; it is simply wall mounted with supplied double sided adhesive; and it is battery operated (battery supplied) and has a full 12 month manufacturers' guarantee. Reader response number 62

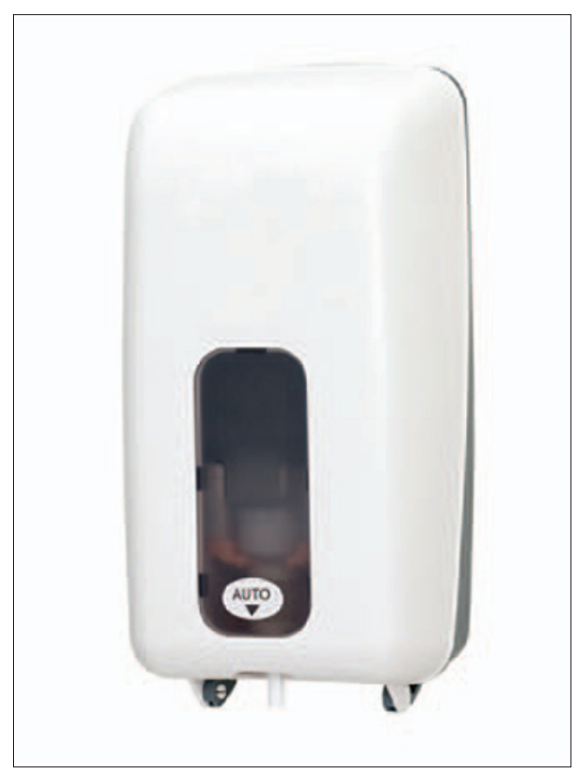

\title{
PROFESSIONAL HARD SURFACE CLEANER
}

Alkaspray-Ultra is specifically formulated for use within the medical/ dental environment to both clean and disinfect all non-pervious hard surfaces in one simple operation with an alcohol free formula.

Alkaspray-Ultra formulation contains highly effective cleansing agents to thoroughly clean hard surface areas while its unique disinfectant properties achieve a high level of disinfection in one operation. It is bactericidal, tuberculocidal, virucidal, yeasticidal and fungicidal providing protection within one minute.

The solution is ready for use on virtually any non-porous surface, medical equipment, furniture and fittings including those surfaces sensitive to solvents/alcohol.

Alkaspray-Ultra is available in both a 1 litre bottle with trigger dispenser and 5 litre economy drums.

Reader response number 63

\section{STERILE PROCEDURE PACKS}

General Medical offer a range of sterile procedure packs containing high quality disposable gowns, drapes and accessories to meet all requirements.

The Universal Pack for minor oral surgery and periodontal procedures offers excellent value for as little as $£ 8.50$ per pack. The Basic Implant Pack contains everything for implant procedures and more advanced

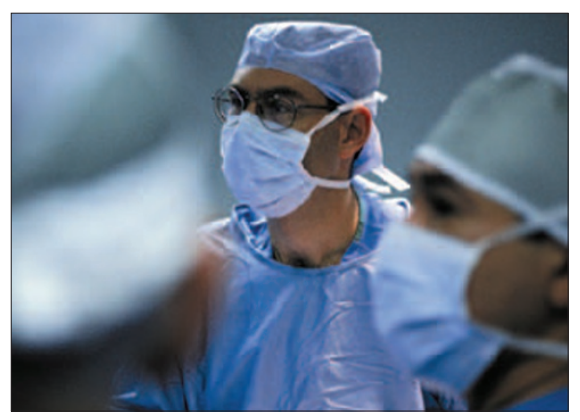

oral surgery and periodontal cases for as little as £21.10 per pack. The De-luxe pack contains a full range of components for all procedures and is similarly competitively priced.

General Medical can also offer custom packs to individual specifications at excellent prices. They enable surgeons to specify the precise combination of disposable gowns, drapes and accessories to meet their individual requirements and save a considerable amount of money.

All General Medical components are available as separate items including gowns, drapes, plastic covers, masks, hats in various styles, suction tubing and tips plus much more. They are detailed in General Medical's Implant Accessory Catalogue.

Reader response number 64

\section{COMPRESSED AIR MEMBRANE DRYER}

The new range of Hi-DRI compressed air membrane dryers from Hi-line Industries have been re-engineered to be technologically superior products, achieving new standards for reliability, performance and energy efficiency. They feature an optimised design combining efficient air flow with high contact areas to maximise the drying performance within a compact unit size. These products are ideal for in-line installation to ensure the delivery of dry compressed air at the point of use. This ensures maximum effectiveness with virtually any dewpoint or degree of drying being achievable.

The Hi-DRI air dryer makes it easier and less expensive to supply pneumatic equipment and instrumentation with clean, dry compressed air. This helps eliminate condensation, oxidation and microbial growth, resulting in reliable and predictable performance of downstream air operated equipment. It also reduces component failures, improves equipment performance and operator satisfaction while showing savings on both warranty and production costs.

These dryers do not contain moving parts and there are no desiccants or refrigerant to replace. They are also compact and lightweight, do not incorporate valves, drains or electrical connectors and do not require adjustments.

With in-line connection ports at each end, the dryers can be easily integrated into an existing airline. Where cost effective centralised refrigeration drying is required, a Hi-line CDA dryer connected directly to a compressor can be combined with individual Hi-DRI units of varying outputs fitted at points of use.

Reader response number 65 